\title{
Ellä toledoth.
}

Von Professor Dr. K. Budde in Marburg.

Mit Spannung werden alle Beteiligten Heinrich Holzingers Anzeige des letzten Buchs von RUdOLF SMEND „Die Erzählung des Hexateuchs" (Theol. Literaturzeitung II. 4. I9I4) in die Hand genommen haben; ich wenigstens weiß nur einen, dessen Urteil ich noch lieber erfahrén hätte als das seinige. Jeder wird sich gefreut haben über das reiche Maß der Anerkennung, die hier dem viel zu früh von uns Geschiedenen gezollt wird; es ist in der Tat ein. sehr starkes Zeugnis für die dem Buche innewohnende Kraft, daß es einen so gediegenen Kenner und nüchternen Kritiker wie HoLZINGER. soweit von seinen eigenen früheren Überzeugungen hat herüberziehen können. Aber gerade daß dem Verfasser dieser wohlverdiente Tribut nun gezollt ist, mag doch auch denen den Mund auftun, die um der Sache willen Fragen und Bedenken zu äußern haben, wie sie HolzINGER selbst, freilich nur Einzelheiten gegenüber, voraussieht. Ich glaube in der Tat, daß es dringend notwendig ist dem Eindruck vorzubeugen, als wenn in SMENDs Buch das letzte Wort der „WELLHAUSENschen Schule“ gesprochen wäre, so daß man mit ihr fertig wäre, wenn und wo man SMEND widerlegt hätte. Dem stand bisher das Bewubtsein im Wege, dab der Tod dem Verfasser die Möglichkeit der Verteidigung seiner Ansichten geraubt hat; jetzt aber dürfen wir sicher sein, daß der denkbar befugteste Anwalt für ihn eintreten wird, wo immer wir ihm unbewußt Unrecht tun oder seine Meinung falsch auslegen sollten. So möchte ich es verstanden wissen, wenn ich hier nur auf einem kleinen Gebiete SMENDs Aufstellungen entgegentrete und etwa noch auf einige weitere Punkte gelegentlich hinweise.

Verhältnismäßig wenig, meint $H$., verschiebe sich bei SMEND das Bild von $P$, wenn auch der geschichtliche Rahmen dieser Kultgesetzgebung 'stark entlastet werde. Ich fürchte, das geschieht weit über das zulässige $\mathrm{Ma}^{-}{ }^{-}$hinaus, derart, daß für den Begriff „Pg“, dên HOLZINGER

Zeitschr. I. d. alttest. Wiss. Jahrg. 34. 1914 
zur Erläuterung von $\mathrm{P}$ beifügt, kaum mehr Platz bleibt. Es ist sehr zu beklagen, daD SMEND seiner Kritik nirgends das Experiment zur Seite gestellt hat, hier eine zusammenhängende Übersetzung dessen, was von $P$ nach seiner Meinung übrig bliebe ${ }^{x}$. Sofort würde sich gezeigt haben, wie sehr $\mathrm{P}$ durch seine Ausscheidungen an innerem Halt und Standfestigkeit verliert, und $d a b$ solchen Resten gegenüber die Theorie eines MAYBAUM und DAHSE von bloßen Zusätzen einer überarbeitenden Redaktion unbedingt Recht behalten würde. Das müßte man natúrlich hinnehmen, sehr gegen SMENDs eigene Úberzeugung und Absicht, wenn die Gründe für seine Ausscheidungen durchschlagend wären; aber das ist keineswegs der Fall. Natürlich weib er so gut wie alle andern, daß wir es mit einem künstlichen, durch vielfache Überarbeitung zustande gekommenen Quellenmosaik zu tun haben, das unweigerlich zwischen den voneinander gelösten Bestandteilen derselben Quelle Widersprüche einerseits, Lücken anderseits aufweisen muß. Daraus erwächst uns natürlich die Aufgabe, wo immer wir dem ursprünglichen Zusammenhang einer Quelle, hier alsó $\mathrm{P}$, nachgehn, für jene Widersprüche und Lücken die Gründe aufzuweisen, und dann den ursprünglichen Bestand nach Möglichkeit herzustellen. Beides aber unterläßt SMEND nicht nur selbst, sondern er benutzt auch viel zu selten, was bereits von anderen dafür getan ist ${ }^{2}$. Vielmehr führt ihm alles nur auf denselben Schluß, Zugehörigkeit zu der letzten Bearbeitung des. Hexateuchs, d. h. den „Zusätzen und Änderungen, von denen die Kompilation des RP (=JED+P)

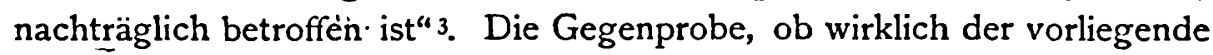
Tatbestand sich aus dem Eingreifen dieser Bearbeitung einleuchtend begreifen läßt, wird wiederum fast durchgängig nicht angestellt. Besonders unglücklich scheint mir nun diese Lösung für die Chronologie der Genesis und des Hexateuchs, die Smend S. I I ff. P abspricht. Denn wenn für irgend etwas, so ist für sie Geschlossenheit und Folgerichtigkeit die

Igl. Wellhausen, Prolegomena (5. Aufl. 1899) S. 33I ff. oder Bacon, Genesis of Genesis oder die Kommentare von Gunkel and ProcksCH.

2 Als Beispiel diene der Ansto $B$ an den Zeitangaben Gen $12{ }_{4}$ b $37=41{ }_{46}$ a S. 12.

3 SMEND fährt fort: „Ich fasse damit hier aber auch solche Änderungen und Zusātze zusammen, die möglicherweise schon $R P$ an JEDP vornahm, ferner solche, die $P$ vielleicht schon vor seiner Vereinigung mit JED erfahren hatte." Er rechtfertigt dieses Verfahren mit der Schwierigkeit und der untergeordneten Bedeutung der Unterscheidung dieser drei Kategorien. Bedauerlich bleibt es, daß der Versuch der Unterscheidung nicht gemacht ist; denn natürlich ist RP oder gár Px etwas ganz andres als eine Interpolation des fertigen JEDP. Selbstverständlich kann der Kritiker nicht nachholen, was der Verfasser unterlassen hat; immerhin dürfte, was hier besprochen wird, nach SMENDs Meinung weit überwiegend der letzten der drei Möglichkeiten zufallen. 
unerläßliche Bedingung. Daß darin Brüche eintreten mußten, wenn die Chronologie $\mathrm{P}$ gehört hatte und nun mit den Bruchstücken dieser Quelle durch einen Redaktor einer Kompilation eingefügt wurde, versteht sich ganz von selbst; wundern kann man sich nur, daß sich davon so wenig aufweisen läßt. Dem letzten Bearbeiter des Hexateuchs aber, wie SMEND selbst ihn nennt, kam doch niemand mehr ins Gehege; er war also in der Lage, seine Chronologie überall unmißverständlich und widerspruchslos einzufügen, so daß ihm gegenüber alle von SMEND gerügten Unstimmigkeiten gar keine Erklärung zulassen. Man fragt sich vergebens, wie SMEND glauben kann, durch die Zuweisung an diesen letzten Redaktor die von ihm geltend gemachten Anstöße erklärt zu haben, so daß es eines Versuchs zu ihrer Lösung darüber hinaus gar nicht mehr bedürfte.

Auch die allgemeinen Gründe für Ausscheidung der Chronologie, der Schöpfungsära, scheinen mir wenig oder nichts zu beweisen. Eine Weltära habe zur Voraussetzung die Chronologie einer nationalen Geschichte, in die sie auslaufe, $\mathrm{P}$ aber habe die Geschichte nur bis zur Verteilung des Landes Kanaan fortgeführt und sei erst nachträglich in den Zusammenhang von Genesis-II Regum (JED) eingefügt worden, der von Judicum an die Geschichte Israels in chronologischer Disposition erzähle. Also könne die Weltära des Hexateuchs frühestens von RP stammen ${ }^{2}$. Wenn aber $P$ den ferneren Geschichtsverlauf nicht neu bearbeitet hat, so hat er doch die alte Bearbeitung JED gekannt, und aller Wahrscheinlichkeit nach hat er sie als genügend und maßgebend anerkannt, da doch auch ihm die Führung Israels durch Jahwe in seiner Volksgeschichte weiterlief. Wollte er nun den Anfang bis zu Josuas Tode durch eine bessere Fassung ersetzen, so mußte er ihn durchaus auf seine Fortsetzung in jenem Werke zupassen. Und hatte er, abgesehen von dem Kern der Gesetzgebung, viel mehr zu streichen als Neues zu bieten, so mubte es ihm nur im höchsten Grade willkommen sein, dab er wenigstens eine grobe Lücke ausfüllen konnte, indem er der Zeitrechnung der Volksgeschichte in Jdc bis Reg ihre feste Grundlage in einer Weltära schuf. Freilich meint SMEND kurz vor jener Stelle, neben den hochernsten gesetzlichen $Z$ wecken, die $P$ verfolge, nehme sich dies künstliche Zahlenspiel sonderbar aus. Ob sich hier wohl der letzte Grund der Abneigung SMENDs gegen die Weltära auftut, eine idealistische Vorstellung von den $Z_{w e c k e n ~}$ s, begründet auf eigene

$\therefore$ So S. 14. 
ästhetische Urteile oder Vorurteile ${ }^{1}$ ? Man sollte denken, $\mathbf{P}$ brächte gerade in seiner Gesetzgebung einen ausreichenden Vorrat an handfestem opus operatum und hölzernem Mechanismus zur Stelle, um gegen die Zumutung übertriebenen Zartgefühls lauten Einspruch zu erheben. Und was wissen wir, wie tiefe Geheimnisse er in seiner Weltära bergen mochte? ${ }^{2}$ Jedenfalls zeigen die mehrfachen Überarbeitungen seiner Weltära, die SMEND (S. I2 I4) sehr mit Unrecht ebenfalls für ihren späten (das will doch sagen redaktionellen) Ursprung geltend macht, dab gerade diese Bereicherung der älteren Überlieferung vor der Nachwelt ihr Glück machte und als Hülle für immer neue Geheimnisse benutzt wurde. - Es wird im ganzen dabei bleiben müssen, dab, je mehr etwas im Geleise des Schemas sich bewegt, um so mehr es dem Geiste der Quelle $\mathbf{P}$ entspricht und ihren eigensten Stempel an sich trägt. So bin ich auch nach wie vor der Überzeugung, daß das Sechstagewerk samt Gottes Ruhen am Sabbath, obwohl es auf die Achtwerkeschöpfung erst nachträglich aufgesetzt ist, doch nicht erst der Redaktion oder SMENDs letzter Bearbeitung (vgl. SMEND S. 9) angehört, sondern von $\mathrm{P}$ selbst seiner aus $\mathrm{J} 2$ stammenden Vorlage einverleibt ist ${ }^{3}$.

$\mathrm{Zu}$ dem Schema, in dessen feierlicher Handhabung die Eigenart von $\mathrm{P}$ recht eigentümlich gipfelt, gehört auch die Hauptstücküberschrift

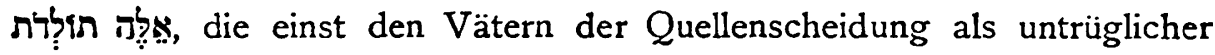
Wegweiser für die Aufdeckung der Quelle $\mathrm{P}$ gedient hat. Nach SMEND (S. I4) ist sie "wenigstens an den meisten Stellen jungen Ursprungs“4.

- Zam ersten gehört auch der Ausspruch S. Io, dem P dürfe das Interesse des Ahnenkultus, das in Gen 23 zum Ausdruck komme, kaum zugetraut werden, zum zweiten der Anstoß an der unmittelbaren Aufeinanderfolge der Zahlenangaben in $16 \times 6$ und $17 \mathrm{x}$.

2 Ich brauche di $\epsilon$ Versuche, auch diesen Geheimnissen nachzudenken, hier nicht aufzuführen.

3 Vgl. mein Buch Die biblische Urgeschichte, 1883, S. 487 ff. Schon DE WetTe (Beiträge z. Einl. in d. A. T. II, I807, S. 35) begegnete der Beobachtung von GABLER, ZIEGLER, ILGEN mit dem Widerspruch: „Unserer Ansicht nach ist diese Bestimmung nach Tagen und die Sabbathruhe notwendig, und im Geiste des Ganzen gegründet.“ In 2 lies nicht mit den Vss.

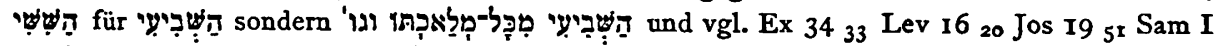
I0 $x_{3}$ II 6 s8. Der Ausfall erklärt sich ohne Mühe. Vielleicht darf man dann den ganzen Schluß des Verses von מכל-מלאכת an als falsch eingerückte Randberichtigung streichen.

4 S. 15 heißt es ,überhaupt oder wenigstens an den meisten Stellen von einem Interpolator eingetragen“, S. 16 wieder „an vielen Stellen von einem Glossator“, der sie möglicherweise "an einzelnen Stellen schon vorfand. Wie sich zeigen wird, hat schon RP sie 36 in JE gelesen.“ Den Beweis dafür bleibt SMEND S. 90 ff. schaldig, da er die Formel dort gar nicht erwähnt ind, was er ausführt, nur für den Inhalt von v. $g$ ff. gilt. Wieder läbt sich das Experiment vermissen, das solch unsicherem Schwanken am besten gesteuert haben würde. 
Nun stammt die Formel jedenfalls überall von der gleichen Hand, und in ihr nicht $P z u$ sehen, dafür müßten starke Gründe beigebracht werden, wie denn Smend selbst zugesteht, daß der Schein für $\mathrm{P}$ spricht. Dagegen entscheidet ihm die schlechte Durchführung des Schemas. Richtig gebraucht im Sinne "dies sind die Nachkommen des und des" $x$ sei die Formel 5 I 10 I I I I0 II 2725 I2 36 I 9 Num 3 I Ruth 4 I8. Diese Bedeutung passe nicht 69 , wo zunächst eine Charakteristik Noahs folge, ferner 25 19, wo zunächst von Isaaks Vater Abraham, dann von seiner Heirat mit Rebekka und deren anfänglicher Unfruchtbarkeit die Rede sei, und 372 , wo die Nachkommen Jakobs längst (35 23-26) aufgezählt seien. In Gen 24 könne unter den Tholedoth des Himmels und der Erde nur deren Entstehung verstanden werden ${ }^{2}$. Stammte die Formel von $P$, so mübte sie überall dieselbe Bedeutung haben und sich mit dieser Bedeutung auch überall in den Zusammenhang fugen. So SMEND. Die Berechtigung dieser Folgerung verstehe ich nicht. Daß überhaupt so verschieáener Gebrauch nicht auf dieselbe Hand zurückgehn könnte, das möchte man schließen; aber daß dies SmENDs Meinung nicht ist, beweist die Fortsetzung: „Man kann auch nicht annehmen, daß die Formel 24692519372 von anderer Hand stamme als an den übrigen Stellen. Denn keinenfalls kann man die Tholedoth Noahs, Isaaks, Jakobs 692519372 von den anderen trennen." Also nur P insbesondere hält er solcher Varïerung nicht für fähig, obgleich er Starrheit des Ausdrucks oder höchste Genauigkeit in der Verwendung der termini sonst nicht als dessen unterscheidendes Merkmal gegenüber anderen Händen geltend macht. Indessen haben wir ja Stoff genug, an dem sich solche Voraussetzung prüfen läßt. In I 5 nennt Gott selbst das Licht im Gegensatz zur Finsternis Qr'; aber in dem gleichen Verse noch braucht der Erzähler dasselbe Wort für den Tag von Morgen zu Morgen, der die Finsternis $=$ Nacht einschliebt. SMEND würde Einspruch erheben, da er ja das Sechstagewerk, und damit auch v. 5b ausscheidet. Aber in

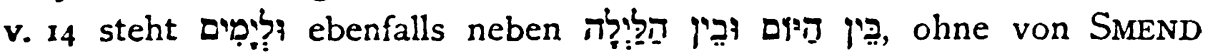
beseitigt zu werden. In I to nennt Gott selbst das Trockene im Gegensatz zum Wasser ארֶ: in v. 1214 a heißt ebenso die ganze

2 "Dies ist der Stammbaum" scheint mir die zutreffendste Übersetzung der Grundbedeutung 2u sein; alle Schattierungen der Bedeutung lassen sich wohl am besten unter "Dies ist die Geschichte“ zusammenfassen, doch ist im Grunde nicht einzusehen, warum wir im Deutschen nicht ebenso kūhn sein sollten wie der Verfasser im Hebräischen, also überall. bei "Stammbaum" bleiben. So Guniker.

2 Die Reibenfolge ist von mir hergestellt. 
Erdenwelt, die irdischen Meere eingeschlossen, im Gegensatz zum Himmel, und in I 2, ehe es einen Himmel gibt, die ganze Masse des Weltstoffs, das Chaos neben Gott. Diese Beispiele dürften genügen, um die Wandlungsfähigkeit des Schriftstellers $\mathrm{P}$ im Gebrauche des Sprachstoffs, der ihm zu Gebote steht, zu beweisen. Wenn er nun der Gesetzgebung eine Vorgeschichte von äußerster Knappheit geben wollte, deren einzelne Abschnitte zum großen Teil bloße Stammbäume bilden mußten, was ist dann begreiflicher, als da sich ihm die Überschrift, die für die meisten Hauptstücke die gewiesene war, in ihrer Bedeutung so lange wandelte und reckte, bis sie sich allen anschmiegte? Wenn er endlich sogar die Kühnheit gewann, die Geschichte der Schöpfung diesem Generalnenner unterzuordnen, so bahnte dazu den Weg sicherlich nicht zum wenigsten die Abstufung ihres Verlaufs in dem Sechstagewerk mit dem Sabbath als Abschluß, schematisch gleichmäßig durchgeführten Abschnitten, die sich recht wohl mit den Generationsstufen des nächsten Hauptstücks Gen 5 vergleichen ließen. SMEND sagt freilich (S. 16), die Auskunft, daß 24 a ursprünglich vor I I gestanden habe, sei mehr als bedenklich. Aber Gründe dafür führt er nicht an, sagt uns auch nicht, was in aller Welt die "letzte Bearbeitung" veranlabt haben könnte, diese Überschrift neu als Unterschrift an einer Stelle einzuschieben, die ihrer so wenig bedurfte ${ }^{\mathbf{x}}$. Was er, mit einem „Dazu kommt" eingeleitet, folgen läßt, daß nämlich 5 I 2 eine Rekapitulation seien, die sofort hinter 23 (in der gesonderten Quelle P natürlich) kaum denkbar sei, vielmehr den Einschub von $24 \mathrm{~b}-426$ vorauszusetzen scheine, das gilt gar nicht gegen 24 a, sonderr

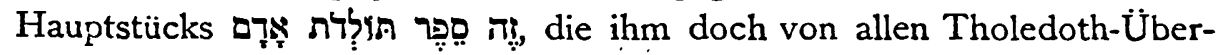
schriften als eine der sichersten erscheint. Denn setzen 5 If. das Stück $24 b-426$ vor sich voraus, so sind sie frühestens von $R p$ verfaßt, nach SMEND sicherlich erst von der letzten Bearbeitung. HolzINGER, der zuerst an dem Text von 5 × 2 gerüttelt hat, ist vorsichtiger; er läbt die Úberschrift v. Ia ganz unangetastet und hält es sogar für möglich, daß an Stelle von v. Ib 2 eine kürzere Notiz über die Erschaffung Adams stand.

Es handelt sich um eine grundlegende Tatsache, deren Verständnis HOLZINGER nur hier vermissen läßt, SMEND überall. Ich darf sie in die-

I Diese Lücke dürfte am besten in Holzingers Kommentar zur Genesis ausgefüllt werden, freilich bin ich nicht imstande, seine Gründe als beweisend anzuerkennen. Nur ob

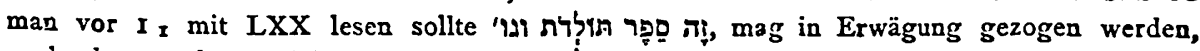

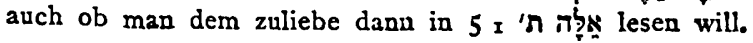


selben Worte kleiden, mit denen ich sie vor einigen Jahren bereits festgelegt habe . „Es ist die Gewohnheit dieser Quelle (P), das neue Hauptstück nach der Überschrift mit einer Wiederholung des in Betracht kommenden Ausschnitts, des passus concernens, wie die Juristen zu sagen pflegten, aus dem vorherigen Hauptstück einzuleiten. So zuerst in 5 I ff., wo v. 1 b 2 die Erschaffung des ersten Menschen aus der Schöpfungsgeschichte Kap. I auszüglich wiederholen.“" Diese Gewohnheit hängt natürlich mit der scharfen Scheidung der Hauptstücke und der neuen Uberschrift eines jeden eng zusammen; jedes von ihnen wird damit vollends auf eigene Füße gestellt. $\mathrm{Ob}$ dadurch soeben erst Gesagtes fast wörtlich wiederholt wird, tut gar nichts zur Sache, so 532 in 6 10, II 26b in 27a. An beiden Wiederholungen stöht sich SMEND S. 13 f. und meint, dem würde durch Beseitigung der sogenannten Zeugungsjahre, die er mit der Weltära der letzten Bearbeitung zuweist, abgeholfen. Wenn er uns. nur am Experiment gezeigt hätte, wie er sich das denkt. Die Zeugung selbśt müßte er doch mit dem Mindestausdruck eines iby'! beibehalten, dann aber bliebe ja in 532 wie in 1 I 26 genau das stehn, was in den nächstfolgenden Versen von $P$ wiederholt wird, so daß die Wiederholung nur noch greller hervorstäche. Ganz diesen Stellen entsprechend muß es auch 10 I hinter „Dies ist der Stammbaum der Söhne Noahs" heißen: „Die Söhne Noahs waren Sem, Ham und Japhet", obgleich das schon zweimal, 532 und 6 ro, gesagt ist; so 2512 von Ismael „Sohn Abrahams, welchen Hagar die Ägypterin, die Magd Saras, dem Abraham geboren hatte“, trotz 1615 f.; so 2519 "Abraham zeugte den Isaak" trotz des Anteils von $\mathrm{P}$ an $2 \mathrm{I} 2$ und der breiten Fortsetzung in 21 3-53. Ob dieses Gesetz ursprünglich auch nur eine einzige Ausnahme zugelassen hat, mag man billig fragen. Die ersté und immerhin die

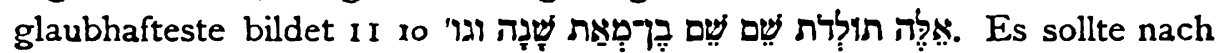
der allgemeinen Regel und nach dem Wortlaut von 2519 insbesondere

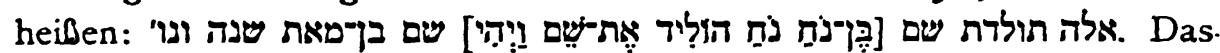
Eingeklammerte kann recht wohl durch Übersehen ausgefallen sein, auch

I ZAW XXX, 1910, S. 277 ff. Seit ich dort (S. 279) die Überzeugung aussprach,

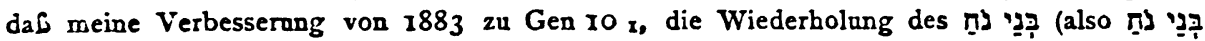

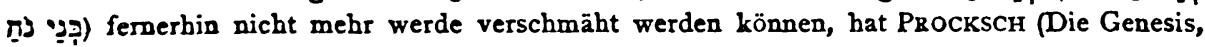
1913) den kleinen Aufsatz zweimal (S. 72 460, lies beidemal „277“ statt „171“) zustimmend angeführt, die Verbesserung aber nicht vollzogen, auch nicht erwähnt.

א für $E$ Ex in V. I nach 127.

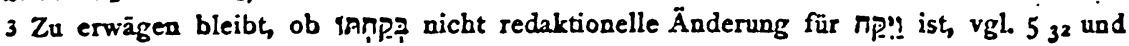
alle itsolichen Stellen bei $P$. 
ein Redaktor möchte es so dicht hinter 10 I gestrichen haben; daneben soll die Möglichkeit anerkannt werden, daß $\mathrm{P}$ hier einmal sich selbst untreu geworden wäre. In Cap. 36 hat ebenso sicher eines der beiden I! Iִ תish von v. I und $9 \mathrm{P}$ gehört ${ }^{2}$, wie jetzt durch die Redaktion der ursprüngliche Wortlaut und Zusammenhang zerstört ist. Bei diesem starken

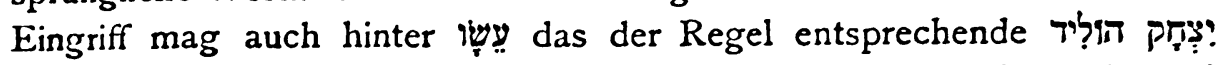
שֶֶ verloren gegangen sein. Das ist sogar sehr wahrscheinlich, weil

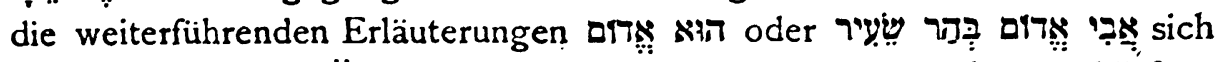
unmittelbar an die Überschrift des Hauptstücks ungeschickt anschließen.

Um Textschäden oder redaktionelle Änderungen handelt es sich endlich mit Sicherheit bei den beiden letzten Stellen, die SMEND (S. 15 f.) unter den sachlichen Bedenken gegen den Gebrauch unserer Formel anführt: „Warum ist II 27 von den Tholedoth Therachs die Rede, obwohl Therach schon v. 32 stirbt, im folgenden nicht aber von den Tholedoth Abrahams? Man meint, die letzteren seien durch Textfehler oder durch Schuld einer Redaktion ausgefallen. Aber diese Auskunft wird abgeschnitten durch den Wortlaut von 25 19: ,Dies sind die Tholedoth Isaaks, Abraham zeugte den Isaak." Danach hat die Überschrift bei Abraham gefehlt, augenscheinlich deshalb, weil sie in der komplizierten Geschichte von Isaaks Geburt nicht so bequem anzubringen war, wie in den anderen Fällen. Ist dem $\mathrm{P}$ diese grobe Ungeschicklichkeit zuzutrauen?" Ich führe die Stelle im ganzen Wortlaut an, um jedes Mibverständnis auszuschließen. - Wer hat wohl bisher jene Überschrift erst bei der Geburt Isaaks (2 I I ff.) 'gesucht? Wellhausen (Composition 3 I899 S. I5) verlangt'sie, sicher viel richtiger, vor $124 \mathrm{~b}$, vielleicht noch mit dem Befehl auszuwandern, der jetzt aus JE gegeben wird. Hier wäre auch irgendwelche Unbequemlichkeit für die Anbringung nicht abzusehen. Was aber der Wortlaut von 2519 gegen ihr früheres Vorhandensein beweisen soll, wird jedem, der meinen bisherigen Ausführungen in der Hauptsache zustimmt, ganz unbegreiflich sein. SMEND weiß aber nichts von der regelmäßigen Wiederholung der grundlegenden Tatsache hinter der Überschrift. Hier scheint er vollends gar nicht zu merken, daß von diesem seinem Einwand keineswegs bloß die Haupt-

I SuEnds Satz über diese Stelle S. 15 „Was sollen neben den Tholedoth der Söhne Noahs 10 I die besonderen Tholedoth Sems II so?" verstehe ich gar nicht, lasse ibn deshalb hier unbesprochen, obgleich er an der Spitze der sachlichen Bedenken gegen die Verwendung der Formel steht.

2 Ich bin nicht der Meinung, daß das andre von $J$ stamme, sondern nehme eine redaktionelle Verdoppelung des einzigen ursprünglichen an. 
überschrift, sondern die ganze Nachricht oder vielmehr Geschichte von der Geburt Isaaks würde getroffen werden, also 21 I ff. nicht nur sondern auch Cap. 17, auf das er so großen Wert legt. Mindestens von der „letzten Bearbeitung“, der er 2519 zuschreibt, müßten diese Abschnitte gestrichen sein, wenn dieser sein Einwand gegen die Forderung von Tholedoth Abrahams zugkräftig wäre. Die richtige Lösung der Schwierigkeit ist sehr einfach. EERDMANS hat sie gefunden, indem er in 1127

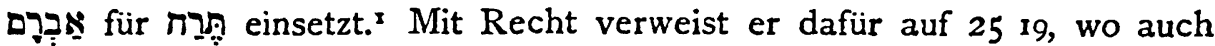
„die ersten Worte der Toledoth [Isaaks!] heißen: Abraham zeugte Isaak, wie II 27 steht: Terach zeugte Abraham usw." Wir sahen, dab der Sache nach die Dinge 5 I 25 12 und in dem hergestellten Wortlaut von Io I (auch II 1o und 36 I oder 9?) genau ebenso liegen. Die einzige Abweichung besteht hier, in I I 27, darin, da von Terach hinter dem passus concernens in v. 3132 noch eigenes Handeln und sein Tod berichtet wird. Das wird in der Tat, falls es sich nicht um ein bloßes

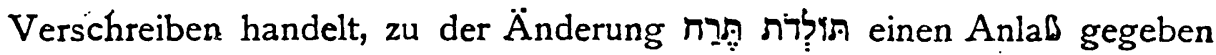
haben, vielleicht neben dem tiefen Einschnitt, den seit der Vereinigung der beiden Quellen die Berufung Abrahams aus $J$ in $12 \mathrm{I}$ darstellte. In $P$ allein aber begreift sich dies Weiterwirken Terachs leicht auch inner-

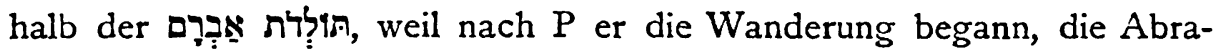
ham fortsetzte und zu Ende führte. ${ }^{2}$

Ich komme zu der letzten Stelle unserer Überschrift in der Genesis, an der SMEND Anstoß nimmt. „Weshalb fehlt", so fragt SMEND S. I6, „die Formel vor Gen 35 23? Statt dessen steht sie 372 vor einem Satz, der, wie oben [vgl. S. 12] bemerkt wurde, jüngeren Ursprungs ist." Merkwürdig, wie man diese beiden Stellen in einem Atem nennen kann, ohne die richtige Lösung sofort zu sehen. Und obendrein hebt SMEND selbst hervor, daß die Aufzählung der Söhne Jakobs in $3522 \mathrm{~b}-26$ an

1 Alttestamentliche Studien I, die Komposition der Genesis, 1908, S. 22. Ob S.MEnd diese Herstellung bei seiner Ablehnung der Annahme eines Textfehlers einschliebt, wird sich kaum entscheiden lassen; ausdrücklich spricht er nur von Ausfall durch Textfehler. Übrigens klingt es bei Eerdayans S. 85 anten fast, als wenn er selbst jene Herstellung wieder vergessen hätte.

2 Bei dieser Gelegenheit mōchte ich den Anstoß beseitigen, den Smend S. 12 an $12{ }_{4} b$ zwischen v. $4^{2}$ aus $J$ und v. 5 aus $P$ nimmt. Vielleicht bin ich der erste gewesen, der diese Schwierigkeit beobachtet hat (Bibl. Urgeschichte 1883 S. 431 f.). Ich lasse dort die Wahl zwischen zwei Lösungen. Entweder bat $v{ }_{4}$ b in der Quelle $P$ hinter v. 5 gestanden, oder der Schluß hat dort im Anschluß an II $_{32}$ gelautet was dan aus Rūcksicht auf $\mathrm{J}$ in v. $\mathrm{x}-4$ abgeändert wäre. Dort gab ich dieser letzten Möglichleeit den Vorzag; aber auch die Umstellung läbt sich aus der Aufnabme von v. $4^{2}$ aus $\mathrm{J}$ recht wohl erlaären, und so mag das die leichtere Annabme sein. 
der falschen Stelle steht. Er sagt (S. 87 f.), sie habe vielleicht in $P$ an früherer Stelle gestanden; das ist WELlHAUSENs Lösungsversuch, der sie (Prolegomenas 1899, S. 333) zwischen 2929 und 3118 einschiebt. Aber hinter 2929 hat $\mathrm{P}$ auch die Geburt der Söhne im einzelnen und die Schließung der Kebsehen erzählt, wovon sich immerhin ausreichende Spuren erhalten haben (vgl. 301 aa. $4 \mathrm{a}, 7$ von is an, $9 \mathrm{~b} 1012222^{\mathrm{I}}$ ); dort ist also die Stelle, die jenes Verzeichnis einnehmen könnte, bereits besetzt. In Wirklichkeit gehört es eben dahin, wo SMEND es S. 15 vermiøt, nämlich hinter die Hauptstücküberschrift in 37 2. Das haben jetzt, wie ich nachträglich sehe, auch SKINNER (mit einem „vielleicht") und PROCKSCH erkannt; nur eins haben sie übersehen, dab nämlich zwischen

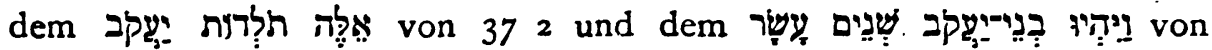

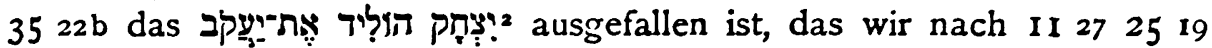
erwarten müssen. Doppelt nötig ist es, weil sonst dem iִיִ die Anknüpfung fehlt. Und wieder ist hinter der Aufzählung $3522 \mathrm{~b}-26$ und vor dem ๆợ von 372 das 'য় zu ergänzen, das wir 2520 an der gleichen Stelle lesen. Daß diesem so weit zurückgreifenden Verzeichnis bei der Vereinigung der Quellen seine ursprüngliche Stelle nicht gelassen wurde, begreift sich leicht. Der Schluß v. 26b wies ihm seine jetzige Stelle, bei der Rückkehr Jakobs aus Paddan Aram; aber nur ohne Hauptstücküberschrift und Vorbericht war es dort zu brauchen. Besondere Beachtung verdient es, daß RP trotzdem die leere. Hülse unserer Formel an der richtigen Stelle, nämlich hinter dem Stammbaum des nicht auserwählten Sohnes Esau (vgl. 2519 mit 25 12), festhielt. Man sieht daraus, einen wie tiefen Eindruck ihm dies feste Schema seiner Grundschrift gemacht hatte3. Wie aber die letzte Bearbeitung der fertigen Genesis dazu hätte kommen sollen, dieselbe leere Hülse, obendrein als neues Gebilde, einzufügen, ist auf keine Weise abzusehen. - Dem allen freilich würde SMEND (vgl. S. 12) entgegenhalten, dab die Zeitangabe in 372 - „und Joseph war I7 Jahre alt" - gar nicht zu P gehören könne. Den Grund für diese Ablehnung gibt er S. 99f., nämlich den Wider-

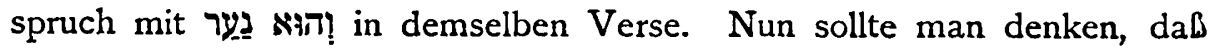
von zwei sich widersprechenden oder stoßenden Angaben die locker sitzende der fester gewurzelten weichen müßte, nicht umgekehrt. In

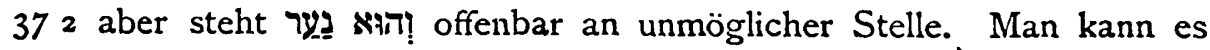

I Nur $30_{4} a$ und $9 b$ schreibt auch SMEND (S. 7I) P zu.

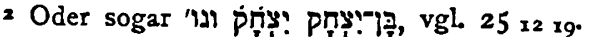

3 Den vorausgehenden Vers 37 × schließt Wellhausen richtig unmittelbar an 368 an; ob er damit in P ebenfalls dicht vor 372 zu stehn kam, mag hier unerörtert bleiben. 
dafür beim non liquet lassen wie WeLlHAUSEN, oder es mit Quellenscheidung versuchen wie DillmanN, HolzingER, KAUTZSCH, oder, wenn man dazu neigt, 372 im ganzen Umfang $P$ zuzuschreiben, mit Text-

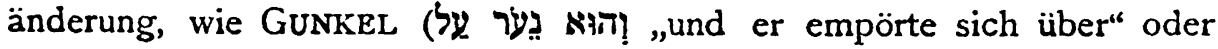
und er verkehrte mit"), SkINNER (unter grundsätzlicher Billigung des ersten Versuchs von GuNKEL, wenn er ihm auch nicht genügt) und PROCKSCH (נָרע nach Prv I I 15 „er war schlecht behandelt“, „er hatte es schlecht"). Jedenfalls läbt sich mit einem so unsichern Textbestandteil der unvergleichlich sichrere der Altersangabe nicht aus dem Sattel heben und gar, als bloße Folgerung daraus, auch noch die nächste in 4I $46 \mathrm{a}$ beseitigen. Vielleicht ist ganz einfach zu helfen. Man übersieht gewöhnlich, daß auch das דָָ unmittelbar hinter der Altersangabe falsch ist ${ }^{x}$, daß es dafür $N i$ ! heißen muß. Die Randberichtigung dieses Fehlers

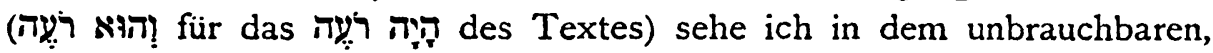
nachträglich wohl oder übel zu einem Bestandteil des Textes zugestutzten

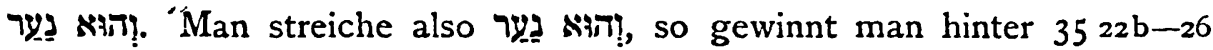

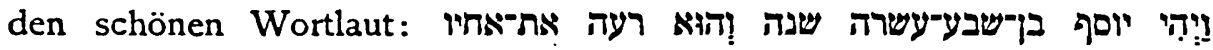
בצהן[-] את-בני בלהה והת־בני ולפה נשי אביו ויבא יוסף ונוי im ganzen Umfang $\mathrm{P}$ gehören kann. ${ }^{2}$

Damit meine ich meinen Gegenstand erschöpft zu haben; ich glaube auf keinen Grund für die Streichung der. Formel aus dem Zusammenhang der Quelle $\mathrm{P}$ die Antwort schuldig geblieben zu sein. $\mathrm{Ob}$ mein Vorschlag überall der richtige ist, darauf kommt wenig an; daß er überall wahrscheinlicher ist als SMENDs Zuweisung an die letzte Überarbeitung, glaube ich beanspruchen zu können. Wir begegneten eben in SMEND einem Arbeiter von starker Eigenart, ja Eigenwilligkeit, der seine Leithypothese rücksichtslos durchsetzt, vielfach so, daß er sich mit dem negativen Ergebnis begnügt, ohne dessen Wirkungen weiter nachzugehn; der wenig nach der Meinung der Vorgänger und Mitarbeiter fragt, oft genug völlig daran vorbeisieht. Durch das ganze Buch hin.wird man der gleichen Eigenart begegnen. Das sollen keine Vorwürfe sein; vielmehr liegt in dieser Anlage SMENDs und dem daraus fließenden Verfahren gerade seine Stärke. Er hätte sein Werk schwerlich zu Ende geführt,

× Es begreift sich wohl als nnberufener Versuch eines Ersatzes für das verlorene

2 Die Erlāaterung des "mit seinen Brüdern“ durch das einschränkende „mit den Söhuen der Bilha und Silpa" will natürlich beschönigend die Schuld auf die halbbürtigen, unedleren Söhne Jakobs abwälzen. Das palt vortrefflich zu P's Anschauungen; aber natürlich steht es daneben frei,"in dieser Apposition eine spätere Hand zu seben. 
er hätte sicher nicht entfernt solche Kraft darin entfaltet, wenn er nicht, ohne rechts oder links zu sehen, dieser seiner Art gefolgt wäre. Wer weil, ob er es uns überhaupt beschert hätte, wenn er sich vorher besser mit der Literatur vertraut gemacht hätte. Sein Held ist doch von Anfang bis zu Ende J2, und SMEND ist augenscheinlich fest überzeugt, zum ersten Mal den Gedanken gefabt und durchgeführt zu haben, daf das Werk dieses bedeutendsten historischen Autors des ganzen Alten Testaments, dieses großen Dichters, durch den vollen Umfang des alten geschichtlichen Schrifttums von der Genesis bis ins Königsbuch durchläuft. Dies Bewußtsein hat ihm sicherlich Mut und Schwung gegeben zu so gewaltiger Arbeit. Nun darf ich hier wohl zuerst eine Berichtigung geben, die mich selbst betrifft. Es ist ein Irrtum, wenn SMEND (S. 22 Anm. 2) mich eine der beiden Schichten, die ich in J nachweise, „auf die Urgeschichte der Menschheit beschränken“ läbt." Das Gegenteil habe ich gegen Ende meines Buchs ausdrücklich festgestellt; ich werde die betreffenden Sätze aus meiner Biblischen Urgeschichte (S. 503) wörtlich hierhersetzen müssen. „Wir haben unsere Untersuchung nur für die biblische Urgeschichte, nur für den ersten Abschnitt der Genesis, geführt. Unsere Ergebnisse haben deshalb nur für diesen Abschnitt Geltung; bezüglich des ganzen übrigen Umfangs der Genesis und vollends des Hexateuchs enthalte ich mich jeder Aussage. In Gen I-I2 5 sind sich J1, J2, J3, Grundschrift und Genesistext, wie oben dargelegt, gefolgt. Dies Ergebnis wird, das hoffe ich zuversichtlich, für die folgenden Abschnitte nicht ohne Frucht bleiben; aber entscheiden kann ich darüber hier nichts." ${ }^{2}$ Ich habe also in meinem Buche von 1883 - und nur von ihm redet SMEND - den zukünftigen Nachweis von $\mathrm{J}_{2}$ über die Urgeschichte hinaus nicht nur für möglich erklärt, sondern als sichere Erwartung ausgesprochen, meinen eigenen Beitrag ausdrücklich als bloße, stofflich bedingte Teilarbeit bezeichnet. Nirgends, soweit ich mich erinnere, habe ich das in den seitdem abgelaufenen dreißig Jahren zurückgenommen, wenn auch die Äußerungen in meiner "Geschichte der althebräischen Literatur" 1906 (S. 103f.) in vorsichtiger Beschränkung auf das Nachgewiesene und das in diesem

× Wenn er vollends sagt „die von ihm angenommene ältere Quellenschrift“", so möchte ich fast in dem Wort „ältere“ einen Druck- oder Schreibfehler sehen. Denn da f mein Jx weiter- und durchlaufe, ist-für meine ganze „Biblische Urgeschichte“ die selbstverstänḋliche Voraussetzung; will man es mit klaren Worten ausgesprochen finden, so vergleiche man S. 403 .

2 Erst hier gesperrt. 
Zusammenhang Entscheidende dahin mißverstanden werden könnten. Soweit also SMEND der Nachweis für das Weiterlaufen von J2 durch den ganzen Hexateuch gelungen ist, ist das nicht gegen meine Erwartung und Vorhersage geschehen, vielmehr bedeutet es deren späte Rechtfertigung. Aber nicht den ersten Versuch dazu. Vielmehr hat fast gleichzeitig mit meiner Biblischen Urgeschichte und unabhängig von meinen Beobachtungen, CH. BRUSTON in Montauban die These SMENDs genau in dem gleichen Umfang aufgestellt und übersichtlich durchgeführt.: Je seltener die Zeitschrift, in der er seine Hauptarbeit veröffentlicht hat, bei uns in Deutschland sich finden wird, umso mehr fühle ich mich verpflichtet, dem verdienten französischen Fachgenossen die gebührende Ehre zu geben. Gelegentliche Vergleiche ergaben mir hie und da ein überraschendes Zusammentreffen in Beobachtungen und Ergebnissen, das für SMENDS Arbeit bei ihrer vollen Unabhängigkeit und 'Selbstàndigkeit den Wert einer bestätigenden Probe hat. Es wird ohne jeden Zweifel lohnen, dem im vollen Zusammenhang nachzugehn. Auch ein Teil des Dankes, den wir SMEND schulden, würde damit abgetragen werden. Denn je weniger man seine Arbeit als abschließend und der Nachprüfung nicht ferner bedürftig ansieht, umso reichere Zinsen wird sein Vermächtnis uns tragen, und umso länger wird auch sein Andenken bei uns in Ehren bleiben.

I Der erste Ansatz in: Les quatre sources des lois de $T$ Exode, I883, 4I Seiten, die Ausführung in: Les deux Jéhovistes, Revue de théologie et de philosophie 1885, P. 3-98. Er führt dort die Scheidung vollständig durch und stellt fest, daß der erste Jahwist mit dem Schisma I Kön I2 abzuschließen scheine, während der zweite sich wahrscheinlich noch über das Schisma hinaus fortsetze. Für die Bücher Richter und Samuel habe ich in dem so betitelten Buche 1890 seine Scheidung nachgeprüft und S. I3jf. 269f. (vgl. auch die erste Erwähnung S. 70) eine Übersicht davon geboten. Von dem Gelingen der Verteilung habe ich mich damals nicht überzeugen können. SMEND hat auch diese Erwähnungen augenscheinlich völlig übersehen und von BkUstons Vorgang nicht die entfernteste Ahnung gehabt.

[Abgeschlossen den 16. Juni 1914] 